\title{
Reopening of an obstructed third ventriculostomy: long-term success and factors affecting outcome in 215 infants
}

\author{
Paul J. Marano, BA, ${ }^{1}$ Scellig S. D. Stone, MD, PhD, ${ }^{1,4}$ John Mugamba, MD, ${ }^{2}$ Peter Ssenyonga, MD, ${ }^{2}$ \\ Ezra B. Warf, ${ }^{3}$ and Benjamin C. Warf, MD ${ }^{1,2,4}$
}

${ }^{1}$ Harvard Medical School, Boston, Massachusetts; ${ }^{2}$ CURE Children's Hospital of Uganda, Mbale, Uganda; ${ }^{3}$ Wheaton College, Wheaton, Illinois; and 4Department of Neurosurgery, Boston Children's Hospital, Boston, Massachusetts

\begin{abstract}
OBJECT The role of reopening an obstructed endoscopic third ventriculostomy (ETV) as treatment for ETV failure is not well defined. The authors studied 215 children with ETV closure who underwent successful repeat ETV to determine the indications, long-term success, and factors affecting outcome.

METHODS The authors retrospectively reviewed the CURE Children's Hospital of Uganda database from August 2001 through December 2012, identifying 215 children with failed ETV (with or without prior choroid plexus cauterization [CPC]) who underwent reopening of an obstructed ETV stoma. Treatment survival according to sex, age at first and second operation, time to failure of first operation, etiology of hydrocephalus, prior CPC, and mode of ETV obstruction (simple stoma closure, second membrane, or cisternal obstruction from arachnoid scarring) were assessed using the Kaplan-Meier survival method. Survival differences among groups were assessed using log-rank and Wilcoxon methods and a Cox proportional hazards model.
\end{abstract}

RESULTS There were 125 boys and 90 girls with mean and median ages of 229 and 92 days, respectively, at the initial ETV. Mean and median ages at repeat ETV were 347 and 180 days, respectively. Postinfectious hydrocephalus (PIH) was the etiology in 126 patients, and nonpostinfectious hydrocephalus (NPIH) in 89. Overall estimated 7-year success for repeat ETV was 51\%. Sex ( $p=0.46$, log-rank test; $p=0.54$, Wilcoxon test), age ( $<v s>6$ months) at initial or repeat ETV ( $p=0.08$ initial, $p=0.13$ repeat; log-rank test), and type of ETV obstruction ( $p=0.61$, log-rank test) did not affect outcome for repeat ETV ( $p$ values $\geq 0.05$, Cox regression). Those with a longer time to failure of initial ETV (>6 months $91 \%, 3-6$ months 60\%, < 3 months 42\%, $p<0.01$; log-rank test), postinfectious etiology (PIH $58 \%$ vs NPIH $42 \%, p=$ 0.02 ; log-rank and Wilcoxon tests) and prior CPC ( $p=0.03$, log-rank and Wilcoxon tests) had significantly better outcome.

CONCLUSIONS Repeat ETV was successful in half of the patients overall, and was more successful in association with later failures, prior CPC, and PIH. Obstruction of the original ETV by secondary arachnoid scarring was not a negative prognostic factor, and should not discourage the surgeon from proceeding. Repeat ETV may be a more durable solution to failed ETVICPC than shunt placement in this context, especially for failures at more than 3 months after the initial ETV. Some ETV closures may result from an inflammatory response that is less robust at the second operation.

http://thejns.org/doi/abs/10.3171/2014.10.PEDS14250

KEY WORDS endoscopic third ventriculostomy; failure; repeat ETV; choroid plexus cauterization; outcome; hydrocephalus; children; Africa; CURE Children's Hospital of Uganda

$\mathrm{I}$ NFANT hydrocephalus is a common cause of death and disability in sub-Saharan Africa, and unlike in more developed countries, the predominant cause is neonatal infection. ${ }^{11-13}$ Shunt dependence is dangerous where timely access to emergency shunt revision surgery is typically not possible. Endoscopic third ventriculostomy (ETV) can be an alternative treatment, but is not successful in the majority of infants younger than 1 year of age. Studies over the past decade at CURE Children's Hospital of Uganda have demonstrated the efficacy of combining ETV and bilateral

ABBREVIATIONS $\mathrm{Cl}=$ confidence interval; $\mathrm{CPC}=$ choroid plexus cauterization; $\mathrm{ETV}=$ endoscopic third ventriculostomy; $\mathrm{HR}=$ hazard ratio; $\mathrm{NPIH}=$ nonpostinfectious hydrocephalus; $\mathrm{PIH}=$ postinfectious hydrocephalus.

SUBMITTED May 20, 2014. ACCEPTED October 15, 2014.

INCLUDE WHEN CITING Published online February 6, 2015; DOI: 10.3171/2014.10.PEDS14250.

DISCLOSURE The authors report no conflict of interest concerning the material or methods used in this study or the findings specified in this paper. 
choroid plexus cauterization (ETV/CPC) in the majority of infants in whom the procedure is successfully completed. ${ }^{8,9,15,16,18-20}$ In response to this, CURE Hydrocephalus, a specialty program within the not-for-profit organization CURE International, was developed to train, equip, and help support neurosurgeons in other developing countries to treat hydrocephalus by ETV/CPC to minimize shunt dependence (www.cure.org/hydrocephalus).

In the subset of patients for whom ETV or ETV/CPC has failed because of closure of the ETV stoma, reopening of the stoma can be performed; however, little is known about the indications for doing so and the likelihood of long-term success. Thus far, results for repeat ETV have been reported in small numbers of patients..$^{2-7}$ To develop guidelines for deciding when to attempt a repeat ETV we needed to know what the efficacy was for this approach as well as any factors that help determine the outcome. In this paper we provide an analysis of repeat ETV efficacy in the largest number of children reported to date, and identify factors that influenced the outcome.

\section{Methods \\ Study Population}

The CURE Children's Hospital of Uganda prospective clinical database was analyzed for the time period of August 2001 through December 2012. A total of 316 patients had undergone repeat ventriculoscopy for failure of prior ETV (with or without CPC) to reopen the ETV if it were found to be obstructed, and 215 (68\%) of these had a technically successful repeat ETV. Patient sex, hydrocephalus etiology (postinfectious hydrocephalus [PIH] or nonpostinfectious hydrocephalus [NPIH]), age at initial ETV or ETV/CPC, time to failure of the initial surgery, and age at second surgery to reopen the ETV were assessed. Nonpostinfectious hydrocephalus included those with myelomeningocele as well as other noninfectious etiologies (such as congenital aqueduct stenosis, congenital idiopathic hydrocephalus, Dandy-Walker complex, and encephalocele). Three patients with reopened ETVs and 2 with abandoned attempts appeared to have posthemorrhagic hydrocephalus, and were also included with the NPIH group. One patient with myelomeningocele also had evidence of severe concomitant ventriculitis associated with the open neural tube defect at the time of the initial ETV and was counted as PIH when assessing the effect of etiology on outcome. The surgeon's observation of the third ventricle and cistern had been recorded prospectively in the database, from which we determined whether the ETV obstruction was secondary to simple closure of the stoma, a second membrane below the floor, or obstruction of the cistern from arachnoid scarring.

\section{Treatment Selection and Operative Technique}

Failure of the initial ETV, as well as failure of a subsequent repeat ETV, was determined as previously reported. ${ }^{8}$ Standard treatment failure criteria, as for a shunt failure, were used to determine whether further surgery was required: a greater than normal growth in head circumference as plotted on a standard growth chart, anterior fontanel distension, new or worsening symptoms of ele- vated intracranial pressure, new or worsening abnormal eye findings, or progressive ventriculomegaly on cranial imaging. Death from any cause was coded as treatment failure. Because there was no access to MRI, it was impossible to determine ETV patency by imaging. In general, ETV failure was treated by primary shunt placement in those with significant prepontine cistern scarring noted at the time of the initial ETV and for those procedures that did not appear to be successful from the very beginning (generally, those failing within 1 month). Those patients with minimal or no cisternal scarring at the first operation who had an initial favorable response to treatment generally underwent repeat ventriculoscopy to inspect the ETV.

The operative technique used for ETV/CPC has been described previously. ${ }^{8}$ The same $3.7-\mathrm{mm}$ flexible steerable ventriculoscope (Karl Storz) was used as for the initial procedure. If the ETV was found to be obstructed, an attempt was made to reestablish the ventriculocisternostomy. If it were found to be patent or if the repeat ETV was not accomplished, a ventriculoperitoneal shunt was typically placed under the same anesthesia. Patients who did not immediately receive a shunt but underwent an alternate new ETV through the lamina terminalis or a CPC alone without being able to reopen the ETV were included along with the other patients categorized as abandoned repeat ETV, and excluded from further analysis.

\section{Statistical Analysis}

Repeat ETV success data were analyzed using the Kaplan-Meier method, and survival differences between groups were assessed using log-rank and Wilcoxon methods. Estimates of hazard ratios (HRs) for the time to repeat ETV failure were derived using a Cox proportional hazards model. A single multivariable model was used to test the effects of several independent categorical variables on success: sex, hydrocephalus etiology (PIH or NPIH), treatment survival according to age at first and second operation ( $<6$ months or $\geq 6$ months), time to failure of first operation ( $<3$ months, 3 to $<6$ months, and $\geq 6$ months), whether CPC was performed at the first operation, and mode of ETV obstruction as noted at the repeat ventriculoscopy (simple closure, simple second membrane, or cisternal arachnoid scarring). Variance inflation factors for significant independent variables were $<1.3$, negating potential concerns for multicollinearity. The appropriateness of the proportional hazards assumption was verified by examining stratified survival curves for each independent variable. Contingency tables were evaluated using Fisher exact tests. Analyses were performed using SPSS Statistics (version 21, IBM Inc.).

\section{Results}

During the study period a total of 316 patients had undergone repeat ventriculoscopy for failure of prior ETV (with or without CPC) to reopen the ETV if it were found to be obstructed. We identified 215 patients (68\%) who underwent technically successful reopening of a closed ETV. In 29 (9.2\%) of the remaining patients the original ETV was found to be patent, and in $72(22.8 \%)$ the ventriculocisternostomy was technically unable to be reestab- 
TABLE 1. Findings at second ventriculoscopy according to etiology

\begin{tabular}{crrccr}
\hline Finding at 2nd Operation & Total & PIH (\%) & Myelomeningocele (\%) & Other (\%) & Total NPIH (\%) \\
\hline ETV reopened & 215 & $126(59)$ & $26(12)$ & $63(29)$ & $89(41)$ \\
\hline Abandoned & 72 & $36(50)$ & $13(18)$ & $23(32)$ & $36(50)$ \\
\hline ETV still open & 29 & $16(55)$ & $2(7)$ & $11(38)$ & $13(45)$ \\
\hline Total & 316 & $178(56)$ & $41(13)$ & $97(31)$ & $138(44)$ \\
\hline
\end{tabular}

lished due to scarring and/or anatomical distortion (abandoned attempts). Table 1 demonstrates these 3 outcomes with respect to the etiology of hydrocephalus. There was no difference between those with PIH and NPIH in regard to the proportion of successful ETV reopening versus abandoned attempts $(p=0.44$, Fisher exact test). That PIH made up 56\% of repeat endoscopies and $59 \%$ of successfully reopened ETVs is completely consistent with the relative incidence of PIH and NPIH for this general population, in which we have previously reported a $60 \%$ incidence of PIH. ${ }^{11}$

Table 2 shows the locations of the first and second ETV. The previously closed ETV in the third ventricle floor was reopened in 205 (95.3\%), either with (41 patients) or without (164 patients) also opening the lamina terminalis. In 5 patients with lamina terminalis ETV only, the previous lamina terminalis ETV had closed and was reopened in addition to creating a new floor ETV. In 4 patients the obstructed lamina terminalis ETV was unable to be reopened but a new floor ETV was created. One patient had lamina terminalis ETV only, which had obstructed and was reopened at the second operation. Two patients had an obstructed floor ETV that was unable to be reopened, but underwent a new lamina terminalis ETV at the second operation when reopening of the floor was abandoned. These 2 operations were counted as abandoned attempts because neither a floor ETV nor a repeat ETV were accomplished.

There were 125 boys and 90 girls. Mean and median ages for initial ETV or ETV/CPC were 229 and 92 days, respectively. Mean and median ages for the repeat ETV were 347 and 180 days, respectively. Mean and median times between first and second operations were 120 days and 68 days, respectively. The overall long-term success rate for repeat ETV was $51 \%$ (Fig. 1A). The median follow-up duration was 17.3 months. There was no difference in repeat ETV success between boys and girls $(\mathrm{p}=0.46$, log-rank test; $p=0.54$, Wilcoxon test; Fig. 1B), and therefore the variable of sex was removed from the model $(\mathrm{p}=$ 0.69 , Cox regression).

The median and mean times from initial ETV at which repeat ETV was performed were 68 and 120.5 days, re- spectively (range 7-2595 days). Only 20 patients underwent repeat ETV sooner than 1 month from the original ETV. Although generally considered a contraindication for repeat ETV in our practice, 9 patients were known to have cisternal scarring noted at the initial ETV.

The ETV was found to be closed in generally 1 of 3 ways: 1) the ETV ostium had sealed over with a thin membrane, at times blending in with the third ventricle floor (or lamina terminalis) so well as to make the original site barely distinguishable; 2) the original ostium was patent, but a second membrane isolated the third ventricle from the cisterns; or 3) the original ETV was obstructed by cisternal arachnoid scarring. In any of these situations, the Bugby wire was used to fenestrate the membranes and reestablish communication between the third ventricle and the cistern.

In cases of ETV/CPC failure, it was noted at the repeat endoscopy that the previously cauterized choroid plexus had become a thin line of scar tissue tracing its original course. Although there were occasional small sprouts or patches of surviving plexus, these appeared to be residual from incompletely cauterized tissue, and there was no suggestion of plexus regeneration.

Figure 1C compares the durability of repeat ETV between the two hydrocephalus etiology categories (PIH and $\mathrm{NPIH})$. There was a significant difference $(\mathrm{p}=0.02, \log$ rank and Wilcoxon tests) in the estimated 7-year success for repeat ETV between those with PIH (58\%) and those with NPIH (42\%), with an HR (adjusted for time to initial failure and performance of CPC) of 0.45 (95\% confidence interval $[\mathrm{CI}] 0.28-0.73, \mathrm{p}<0.01)$.

There was no statistically significant difference in treatment survival between those greater than or less than 6 months of age at the time of the first operation $(p=0.08$, log-rank and Wilcoxon tests; Fig. 1D) or at the time of the second operation $(p=0.13$, log-rank test; $p=0.22$, Wilcoxon test; Fig. 2A), and thus both variables were removed from the model $(\mathrm{p}=0.06$ and $\mathrm{p}=0.43$, respectively, Cox regression).

Figure 2B demonstrates significantly different repeat ETV treatment survival in relation to the time to failure

TABLE 2. Location of initial and redo ETV

\begin{tabular}{lcccccc}
\hline 1st ETV & Redo Floor Only & Redo Floor \& Redo LT & $\begin{array}{c}\text { Redo Floor \& } \\
\text { New LT }\end{array}$ & Redo LT Only & $\begin{array}{c}\text { Redo LT \& } \\
\text { New Floor }\end{array}$ & New Floor Only \\
\hline Floor only & 154 & 0 & 26 & 0 & 0 & 0 \\
\hline Floor \& LT & 10 & 15 & 0 & 0 & 0 & 0 \\
\hline LT only & 0 & 0 & 0 & 1 & 5 & 4 \\
\hline Total & 164 & 15 & 26 & 1 & 5 & 4 \\
\hline LT = Lamina terminalis. & &
\end{tabular}



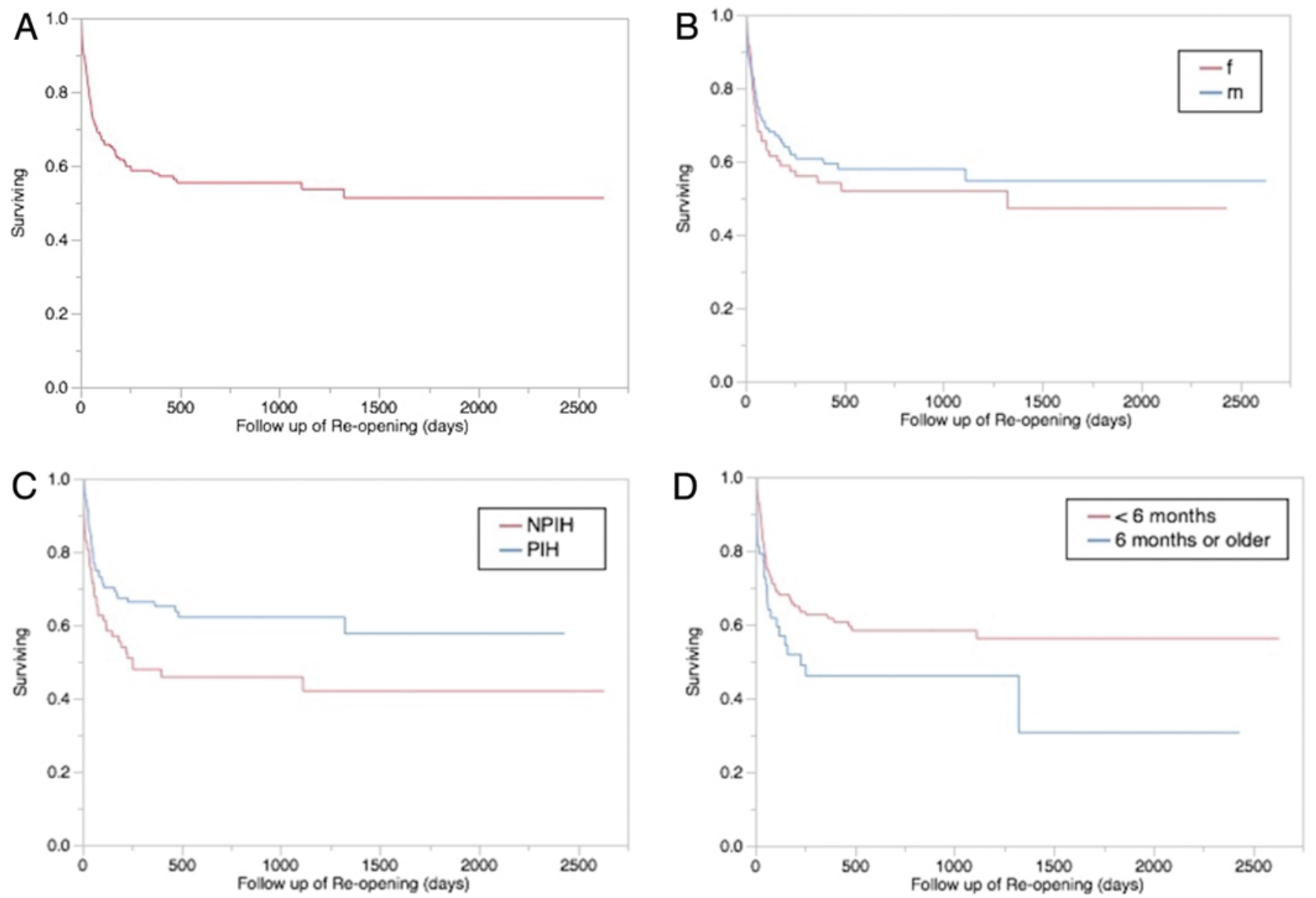

FIG. 1. Line graphs showing overall treatment survival for repeat ETV (A), as well as treatment survival according to $\operatorname{sex}(\mathbf{B} ; f=$ female, $m=$ male), hydrocephalus etiology $(C)$, and age at first ETV (D). Figure is available in color online only.

of the first ETV ( $p<0.01$, log-rank and Wilcoxon tests), which remained significant when analyzed by Cox regression adjusted for hydrocephalus etiology and performance of CPC. When failure of the original ETV occurred after 6 months, repeat ETV had an estimated 7-year success rate of $91 \%$ (HR $0.14,95 \%$ CI $0.03-0.56 ; p=0.01$ ). When failure occurred at 3-6 months or before 3 months, the estimated 7-year success rates for repeat ETV were $60 \%$ (HR 0.53, 95\% CI 0.30-0.91; p = 0.02) and 42\% (reference hazard, $\mathrm{p}<0.01$ ), respectively.

We found no relationship between type of ETV obstruction (simple closure, simple second membrane, or cisternal arachnoid scarring) and outcome ( $p=0.61$, logrank test; $p=0.74$, Wilcoxon test; Fig. $2 \mathrm{C}$ ), and therefore type of ETV obstruction was removed from the model ( $p$ values $\geq 0.57$, Cox regression).

Patients who underwent ETV with CPC as the initial procedure experienced significantly greater success with the repeat ETV than those who did not undergo CPC at any point $(\mathrm{p}=0.03, \log$-rank and Wilcoxon tests; Fig. 2D), with an HR (adjusted for etiology and time to initial failure) of 0.51 (95\% CI $0.31-0.83 ; \mathrm{p}=0.01)$. Only 13 patients underwent ETV only at the first procedure but repeat ETV with $\mathrm{CPC}$ at the second operation, thus preventing any conclusions about this small subgroup.

\section{Discussion}

Endoscopic third ventriculostomy failure (with or without CPC) can occur either because of stoma obstruction or because a functioning ETV simply fails to adequately treat the hydrocephalus. The latter should be evident relatively soon after treatment, whereas failure from ETV obstruction could theoretically manifest at some later point. Even late ETV failure beyond 1 year can occur, although this appears to be rare. ${ }^{1}$ Failure from ETV obstruction may lend itself to treatment by reopening of the ETV, but the indications, efficacy, and factors affecting outcome have not been clear. This series of 215 children undergoing repeat ETV is the largest ever analyzed. The estimated 7 -year treatment survival rate was $51 \%$. Later time to failure, postinfectious etiology, and prior CPC each favored success.

The results for reopening a closed ETV have been previously reported in smaller patient cohorts..$^{2-7}$ Of the 2 reports involving more than 20 patients (the majority with "obstructive" causes of hydrocephalus), the reported success rates for repeat ETV were $75 \%$ (of 40 patients) ${ }^{5}$ and $89 \%$ (of 32 patients). ${ }^{3}$ Peretta and coauthors found subarachnoid adhesions in the cisterns below the floor, as opposed to simple stoma closure, to be a significant negative 

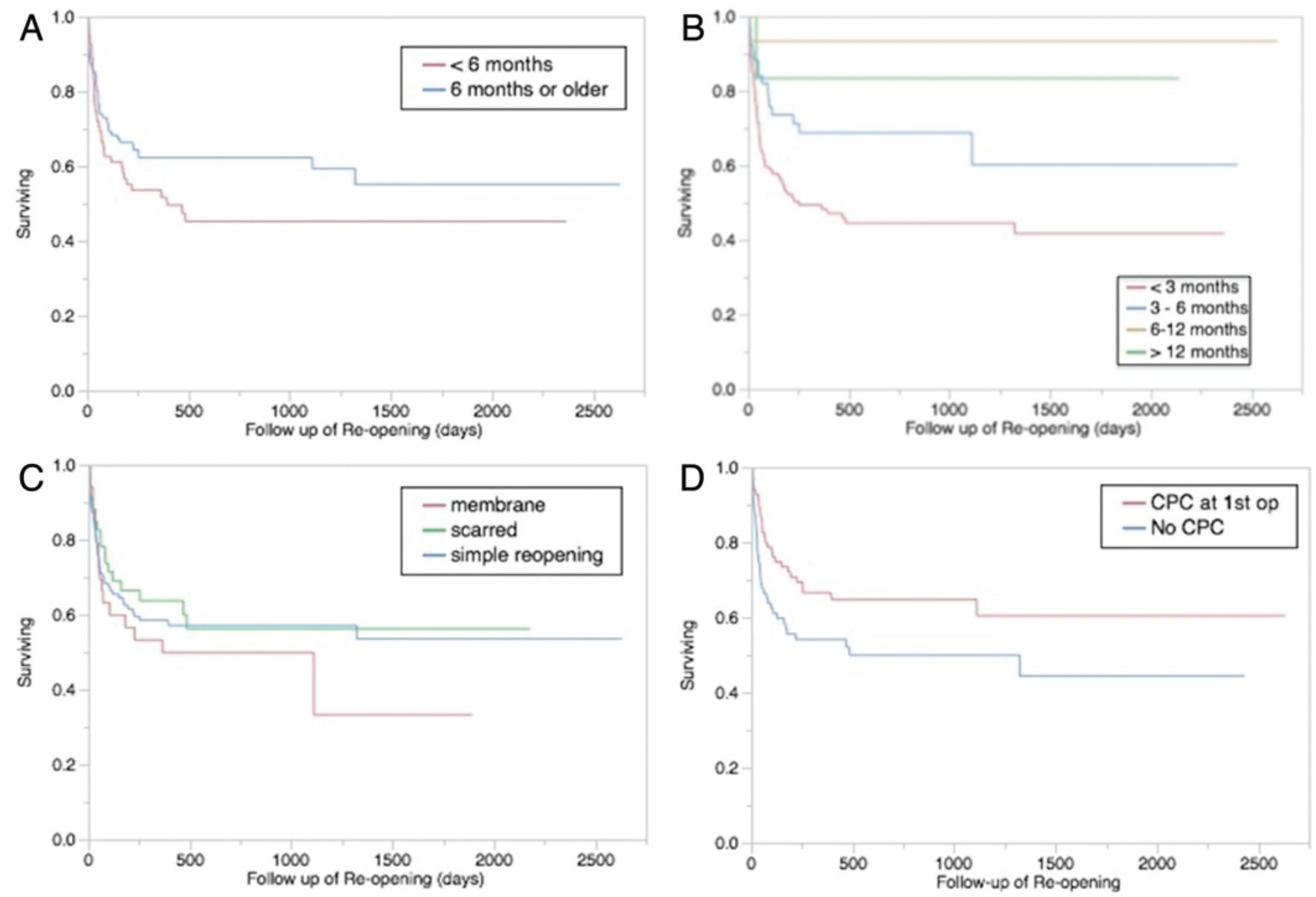

FIG. 2. Line graphs showing treatment survival according to age at repeat ETV (A), time to failure from the original ETV (B), type of ETV obstruction (C), and with and without CPC (D). Figure is available in color online only.

prognostic factor. ${ }^{5}$ These authors found that age less than 2 years at the time of the initial ETV to be a negative prognostic factor as well (50\% vs $90 \%$ success, respectively), but did not evaluate time to failure of the first ETV or age at time of repeat ETV. Mahapatra and coauthors found repeat ETV after "early" failure ( $<7$ days from first ETV) to be a negative factor, with $50 \%$ success in that group but $78 \%$ success in those failing after more than 7 days (mean 1.4 years). ${ }^{3}$ These authors did not find any significant difference in outcome with age at repeat ETV.

Similar to Mahapatra and colleagues, ${ }^{3}$ we found that time to original ETV failure correlated with likelihood of repeat ETV success, although we analyzed time to failure of $0-3,3-6$, and more than 6 months as opposed to a single cutoff point of 7 days. The longer the time to failure, the more likely repeat ETV was to be successful. Those patients with a failed original ETV after 6 months had better than $90 \%$ success with the repeat ETV. Also, like Mahapatra and coauthors, we found no difference in outcome according to the age at the time of repeat ETV, but in contrast to Peretta and colleagues, we found no correlation between outcome for repeat ETV and patient age at the time of the original ETV.

It was not surprising that overall success for repeat ETV was significantly better in patients for whom a CPC had been completed at the first operation, given prior reports that this improves the prospects for ETV success overall in the very young. ${ }^{8-10,15,16,18-20}$ This may help account for the lack of an age effect on repeat ETV outcome.

In contrast to the study by Peretta and colleagues, ${ }^{5}$ we found no significant difference in repeat ETV outcome in association with the type of ETV obstruction. More specifically, we did not find cisternal arachnoid scarring to be a negative prognostic factor, as their study had suggested. We also investigated simple second membranes below the ETV stoma as a distinct group, as opposed to arachnoid scarring, and likewise found no difference from the other two groups (simple stoma closure and arachnoid scarring). Thus, the finding of arachnoid scarring obstructing the stoma should not be a contraindication to dissecting through this into a clear cistern.

The timing and risk factors specific to ETV obstruction are not well characterized, although factors affecting the overall likelihood of ETV success in our Ugandan population have been documented and include age at treatment, hydrocephalus etiology, the status of the prepontine cistern and aqueduct, and the extent of CPC. ${ }^{17,18}$ Risk of ETV closure might be more likely in the context of an inadequate initial ETV (size of stoma or coaptation of redundant tissue at the stoma perimeter), poor flow across the ETV secondary to unfenestrated secondary membranes or cisternal scarring, or a residual active intraventricular 
or subarachnoid space inflammatory response in cases of posthemorrhagic hydrocephalus or PIH. If hydrocephalus in a given patient is adequately treated when the ETV is patent, then failure from its closure could logically be treated by reopening it. At that point, the conditions for long-term patency may have changed. Thus, if arachnoid scarring or stoma closure evolved after the initial ETV secondary to a persistent inflammatory response, the conditions may be more favorable at the time of the repeat ETV. This may help explain why an etiology of PIH (the majority in this population with ventriculitis as opposed to meningitis ${ }^{11}$ ) was not a negative prognostic factor for repeat ETV success in this study. In fact, the outcome for PIH was significantly better than for NPIH $(58 \%$ compared with $42 \%$ ). This being the case, a longer interval between the first and second operations would favor success, as found in the present study. It has also been suggested that young age itself may correlate with a greater tendency toward forming cisternal scarring following ETV?

ETV patency can be assessed by MRI techniques, but in the context of low-middle income countries such as those in which CURE Hydrocephalus has established treatment sites, this is not available. Thus, the status of the ETV can only be reliably assessed with repeat ventriculoscopy. This clinical series from CURE Children's Hospital of Uganda reports the efficacy of successfully reestablishing the ETV when found to be obstructed. When repeat ETV cannot be accomplished or the original stoma is found to be functional, a ventriculoperitoneal shunt is typically placed at the time of the same operation. We have previously demonstrated that there is no increased risk of shunt failure or infection when shunt placement occurs in the context of an abandoned or previously failed ETV or ETV/CPC. ${ }^{14}$ Thus, repeat exploratory endoscopy at the time of shunt placement should not increase the likelihood of subsequent shunt complications or infection.

In limited resource centers where large patient volume can outstrip neurosurgical manpower and resources, task sharing with nonneurosurgeons who are trained to place shunts is sometimes necessary. It becomes especially important in those circumstances to have evidence-based guidelines in place that can optimize treatment selection. We have previously attempted to establish both preoperative and intraoperative criteria that can assist with initial treatment selection..$^{17,18}$ Our aim here was to examine both the efficacy of repeat ETV and what accessible clinical factors might influence outcome.

In this study, the longer the time to failure of the initial ETV, the more likely repeat ETV was to be successful. Repeat ETV was also significantly more likely to succeed when the initial operation had included CPC. The age at primary or secondary ETV did not appear to influence outcome. Success for repeat ETV was more likely for patients with PIH. This finding may suggest that some ETV closures result from an inflammatory response that is less robust at the second operation because of a change in milieu, rather than the effect of age per se. Infants presenting with PIH may have a residual inflammatory response to the original infection that quells with time. Alternatively, younger infants may be intrinsically more capable of healing the stoma. However, this is not supported by the obser- vation that neither the age at the time of initial ETV nor age at the time of repeat ETV affected the outcome for repeat ETV.

\section{Conclusions}

This study demonstrates that, in our sub-Saharan African population, a reopened ETV can remain open longterm in approximately half of the patients overall, and is particularly successful in those patients with later ETV failures, those who underwent CPC, and those with PIH. Obstruction of the original ETV by secondary arachnoid scarring was not a negative prognostic factor, and should not discourage the surgeon from proceeding with reestablishing the ventriculocisternostomy into unobstructed cisternal spaces. Repeat ETV may be a more durable solution to failed ETV/CPC than shunt placement in this context, and should be considered preferable to shunt placement, especially in the context of treatment failures more than 3 months after the initial ETV. In the CURE Hydrocephalus program, we currently recommend repeat endoscopy to assess for ETV obstruction rather than primary shunt placement for all infants presenting with failed ETV/CPC more than 3 months after surgery, unless significant cisternal scarring had been noted at the original operation.

\section{References}

1. Drake J, Chumas P, Kestle J, Pierre-Kahn A, Vinchon M, Brown J, et al: Late rapid deterioration after endoscopic third ventriculostomy: additional cases and review of the literature. J Neurosurg 105 (2 Suppl):118-126, 2006

2. Hellwig D, Giordano M, Kappus C: Redo third ventriculostomy. World Neurosurg 79:S22.e13-S22.e20, 2013

3. Mahapatra A, Mehr S, Singh D, Tandon M, Ganjoo P, Singh $\mathrm{H}$ : Ostomy closure and the role of repeat endoscopic third ventriculostomy (re-ETV) in failed ETV procedures. Neurol India 59:867-873, 2011

4. Mohanty A, Vasudev MK, Sampath S, Radhesh S, Sastry Kolluri VR: Failed endoscopic third ventriculostomy in children: management options. Pediatr Neurosurg 37:304-309, 2002

5. Peretta P, Cinalli G, Spennato P, Ragazzi P, Ruggiero C, Aliberti F, et al: Long-term results of a second endoscopic third ventriculostomy in children: retrospective analysis of 40 cases. Neurosurgery 65:539-547, 2009

6. Surash S, Chumas P, Bhargava D, Crimmins D, Straiton J, Tyagi A: A retrospective analysis of revision endoscopic third ventriculostomy. Childs Nerv Syst 26:1693-1698, 2010

7. Wagner W, Koch D: Mechanisms of failure after endoscopic third ventriculostomy in young infants. J Neurosurg 103 (1 Suppl):43-49, 2005

8. Warf BC: Comparison of endoscopic third ventriculostomy alone and combined with choroid plexus cauterization in infants younger than 1 year of age: a prospective study in 550 African children. J Neurosurg 103 (6 Suppl):475-481, 2005

9. Warf BC: Congenital idiopathic hydrocephalus of infancy: the results of treatment by endoscopic third ventriculostomy with or without choroid plexus cauterization and suggestions for how it works. Childs Nerv Syst 29:935-940, 2013

10. Warf BC: Endoscopic third ventriculostomy and choroid plexus cauterization for pediatric hydrocephalus. Clin Neurosurg 54:78-82, 2007

11. Warf BC: Hydrocephalus in Uganda: the predominance of infectious origin and primary management with endoscopic third ventriculostomy. J Neurosurg 102 (1 Suppl):1-15, 2005 
12. Warf BC: Pediatric hydrocephalus in East Africa: prevalence, causes, treatments, and strategies for the future. World Neurosurg 73:296-300, 2010

13. Warf BC, Alkire BC, Bhai S, Hughes C, Schiff SJ, Vincent JR, et al: Costs and benefits of neurosurgical intervention for infant hydrocephalus in sub-Saharan Africa. J Neurosurg Pediatr 8:509-521, 2011 (Erratum in J Neurosurg Pediatr 9:109, 2012)

14. Warf BC, Bhai S, Kulkarni AV, Mugamba J: Shunt survival after failed endoscopic treatment of hydrocephalus. J Neurosurg Pediatr 10:463-470, 2012

15. Warf BC, Campbell JW: Combined endoscopic third ventriculostomy and choroid plexus cauterization as primary treatment of hydrocephalus for infants with myelomeningocele: long-term results of a prospective intent-to-treat study in 115 East African infants. J Neurosurg Pediatr 2:310-316, 2008

16. Warf BC, Dewan M, Mugamba J: Management of DandyWalker complex-associated infant hydrocephalus by combined endoscopic third ventriculostomy and choroid plexus cauterization. J Neurosurg Pediatr 8:377-383, 2011

17. Warf BC, Kulkarni AV: Intraoperative assessment of cerebral aqueduct patency and cisternal scarring: impact on success of endoscopic third ventriculostomy in 403 African children. J Neurosurg Pediatr 5:204-209, 2010

18. Warf BC, Mugamba J, Kulkarni AV: Endoscopic third ventriculostomy in the treatment of childhood hydrocephalus in Uganda: report of a scoring system that predicts success. J Neurosurg Pediatr 5:143-148, 2010
19. Warf BC, Stagno V, Mugamba J: Encephalocele in Uganda: ethnic distinctions in lesion location, endoscopic management of hydrocephalus, and survival in 110 consecutive children. J Neurosurg Pediatr 7:88-93, 2011

20. Warf BC, Tracy S, Mugamba J: Long term outcome for endoscopic third ventriculostomy alone or in combination with choroid plexus cauterization for congenital aqueductal stenosis in African infants. J Neurosurg Pediatr 10:108-111, 2012

\section{Author Contributions}

Conception and design: BC Warf. Acquisition of data: BC Warf, Marano, Mugamba, Ssenyonga, EB Warf. Analysis and interpretation of data: BC Warf, Marano. Drafting the article: BC Warf. Critically revising the article: BC Warf, Marano, Stone. Reviewed submitted version of manuscript: BC Warf, Marano, Mugamba, Ssenyonga, EB Warf. Approved the final version of the manuscript on behalf of all authors: BC Warf. Statistical analysis: Marano, Stone. Administrative/technical/material support: BC Warf, Mugamba, Ssenyonga. Study supervision: BC Warf.

\section{Correspondence}

Benjamin C. Warf, Department of Neurosurgery, Boston Children's Hospital, 300 Longwood Ave., Boston, MA 02115. email: benjamin.warf@childrens.harvard.edu. 